\title{
Age-dependent increase in the expression of antioxidant-like protein-1 in the gerbil hippocampus
}

\author{
JIN-A PARK ${ }^{1 *}$, JOON HA PARK $^{2 *}$, JI HYEON AHN ${ }^{3}$, JONG-DAI KIM $^{4}$, MOO-HO WON ${ }^{2}$ and CHOONG-HYUN LEE ${ }^{1}$ \\ ${ }^{1}$ Department of Pharmacy, College of Pharmacy, Dankook University, Cheonan, South Chungcheong 31116; \\ ${ }^{2}$ Department of Neurobiology, School of Medicine, Kangwon National University, Chuncheon, Gangwon 24341; \\ ${ }^{3}$ Department of Biomedical Science, Research Institute of Bioscience and Biotechnology, Hallym University, \\ Chuncheon, Gangwon 24252; ${ }^{4}$ Division of Food Biotechnology, School of Biotechnology, \\ Kangwon National University, Chuncheon, Gangwon 24341, Republic of Korea
}

Received February 11, 2016; Accepted July 26, 2016

DOI: $10.3892 / \mathrm{mmr} .2016 .5619$

\begin{abstract}
Antioxidant-like protein-1 (AOP-1) reduces the intracellular level of reactive oxygen species. In the present study, the age-related change in AOP-1 expression in the hippocampus among young, adult and aged gerbils was compared using western blot analysis and immunohistochemistry. The results demonstrated that the protein expression of AOP-1 was gradually and significantly increased in the hippocampus during the normal aging process. In addition, the age-dependent increase in AOP-1 immunoreactivity was also observed in pyramidal neurons of the hippocampus proper; however, in the dentate gyrus, AOP-1 immunoreactivity was not altered during the normal aging process. These results indicated that the expression of AOP-1 is significantly increased in the hippocampus proper, but not in the dentate gyrus, during the normal aging process.
\end{abstract}

\section{Introduction}

Antioxidant-like protein-1 (AOP-1) is an antioxidant protein with $93.3 \%$ homology to SP-22, a mitochondrial antioxidant protein (1). AOP-1, which is expressed in the cytoplasm, as well as in the mitochondria, has been known to reduce intracellular levels of reactive oxygen species (ROS) by co-operating with

Correspondence to: Professor Moo-Ho Won, Department of Neurobiology, School of Medicine, Kangwon National University, 1 Kangwondaehak, Chuncheon, Gangwon 24341, Republic of Korea E-mail:mhwon@kangwon.ac.kr

Professor Choong-Hyun Lee, Department of Pharmacy, College of Pharmacy, Dankook University, 119 Dandae-ro, Cheonan, South Chungcheong 31116, Republic of Korea

E-mail: anaphy@dankook.ac.kr

*Contributed equally

Key words: antioxidant-like protein-1, aging, hippocampus, pyramidal neurons, gerbil mitochondrial thioredoxin and is important in the maintenance of mitochondrial mass and membrane potential (1-3). In addition, AOP-1 is associated with various biological processes, including cell proliferation, differentiation, apoptosis and gene expression (4).

Aging leads to neuroanatomical and neurophysiological alterations in the central nervous system (5-7). In the brain, age-related changes are closely associated with alterations in DNA damage, synaptic vesicle transport, autophagic degradation and neuronal activity (8-12). In particular, oxidative stress, which accumulates with age, is considered to be one of the major causes of aging and age-associated cognitive impairment (8,13-16). Certain previous studies have demonstrated that antioxidant supplementation, which reduces oxidative stress in the brain, ameliorates histopathological alterations, as well as learning and memory deficits in animal models for brain aging (17-19). In addition, it is well established that marked increases in oxidative stress occur in the brain during the normal aging process $(16,20)$. However, age-related changes in AOP-1 in the brain remain to be fully elucidated. Since the hippocampus, which is an important region associated with learning and memory, is the most vulnerable brain region to the aging process (21-24), the present study aimed to investigate age-related changes in AOP-1 in the hippocampus of the Mongolian gerbil, which is a suitable model for research on aging $(20,25)$.

\section{Materials and methods}

Experimental animals. A total of 42 male Mongolian gerbils (Meriones unguiculatus) were obtained from the Experimental Animal Center, Kangwon National University (Chuncheon, South Korea). As previously described (9), the animals were divided into the following three groups ( $n=14 /$ group): Young (3-4 months old; weighing 65-72 g), adult (10-12 months old; weighing 77-83 g) and aged (18-24 months old; weighing 85-95 g). The animals were housed in a conventional state under an adequate temperature $\left(23 \pm 3^{\circ} \mathrm{C}\right)$ and relative humidity $(55 \pm 5 \%)$ with a $12 \mathrm{~h}$ light/dark cycle, and were allowed free access to food and water. All experimental procedures for animal handling and 
use were approved by the Institutional Animal Care and Use Committee at Kangwon National University (approval no. KW-130424-3).

Western blot analysis. To examine alterations in the protein expression of AOP-1 in the hippocampus during normal aging, the animals ( $\mathrm{n}=7 /$ group) were used, and western blot analysis was performed, as previously described $(9,10,26)$. Briefly, the hippocampus was homogenized and centrifuged, at $16,000 \mathrm{x} \mathrm{g}$ for $20 \mathrm{~min}$ at $4^{\circ} \mathrm{C}$ and the supernatants were subjected to western blot analysis. Following centrifugation, the protein level in the supernatants was determined using a micro bicinchoninic acid protein assay kit (Sigma-Aldrich, St. Louis, MO, USA) with bovine serum albumin as a standard (Pierce Biotechnology, Inc., Rockford, IL, USA). Aliquots containing $20 \mu \mathrm{g}$ total protein were boiled in loading buffer containing $150 \mathrm{mM}$ Tris (pH 6.8, Sigma-Aldrich), $3 \mathrm{mM}$ dithiothreitol, $6 \%$ sodium dodecyl sulfate, $0.3 \%$ bromophenol blue and $30 \%$ glycerol. The aliquots were then loaded onto a $12 \%$ polyacrylamide gel. Following electrophoresis, the proteins were transferred to nitrocellulose transfer membranes (Pall Corporation, East Hills, NY, USA). To reduce background staining, the membranes were incubated with $5 \%$ non-fat dry milk (Sigma-Aldrich) in phosphate-buffered saline (PBS) containing 0.1\% Tween-20 (PBST; Sigma-Aldrich) for $45 \mathrm{~min}$ at room temperature. Membranes were incubated with monoclonal mouse anti-AOP-1 (cat. no. A7674; dilution, 1:2,500; Sigma-Aldrich) or monoclonal mouse anti- $\beta$-actin (cat. no. A53161; dilution, 1:2,500; Sigma-Aldrich) overnight at $4^{\circ} \mathrm{C}$. Following washing with PBST three times, the membranes were incubated with peroxidase-conjugated goat anti-mouse (cat. no. sc-2031; dilution, 1:1,000; Santa Cruz Biotechnology, Inc., Dallas, TX, USA) for $1 \mathrm{~h}$ at room temperature. An enhanced chemiluminescence kit (Pierce ECL Western Blotting Substrate; Thermo Fisher Scientific, Inc., Waltham, MA, USA) The result of the western blot analysis was scanned and densitometric analysis for the quantification of the bands was performed using Image J 1.49 software (National Institutes of Health, Bethesda, MD, USA), which was used to count the relative optical density (ROD). The expression of AOP-1 was normalized against that of $\beta$-actin, which was used as the internal control protein. A ratio of the ROD was calibrated as a percentage, with the young group designated as $100 \%$. Western blot analysis was performed with three replicates.

Immunohistochemistry. To examine age-related changes in AOP-1 immunoreactivity in the gerbil hippocampus, immunohistochemical staining was performed, as previously described $(9,10,26)$. In brief, the animals ( $n=7 /$ group) were anesthetized with Zoletil $50(30 \mathrm{mg} / \mathrm{kg}$; Virbac, Carros, France) and perfused transcardially with $4 \%$ paraformaldehyde (Sigma-Aldrich) in 0.1 M phosphate-buffered saline (pH 7.4; Sigma-Aldrich). The brains were postfixed with the same solution for $6 \mathrm{~h}$ and sectioned with a cryostat at $30 \mu \mathrm{m}$. Immunohistochemical staining for AOP-1 was performed using monoclonal mouse anti-AOP-1 (cat. no. A7674; dilution, 1:500; Sigma-Aldrich) as a primary antibody. The brain tissues were subsequently incubated with biotinylated goat anti-mouse immunoglobulin G (cat. no. BA-9200; 1:200; Vector Laboratories, Burlingame, CA, USA) and

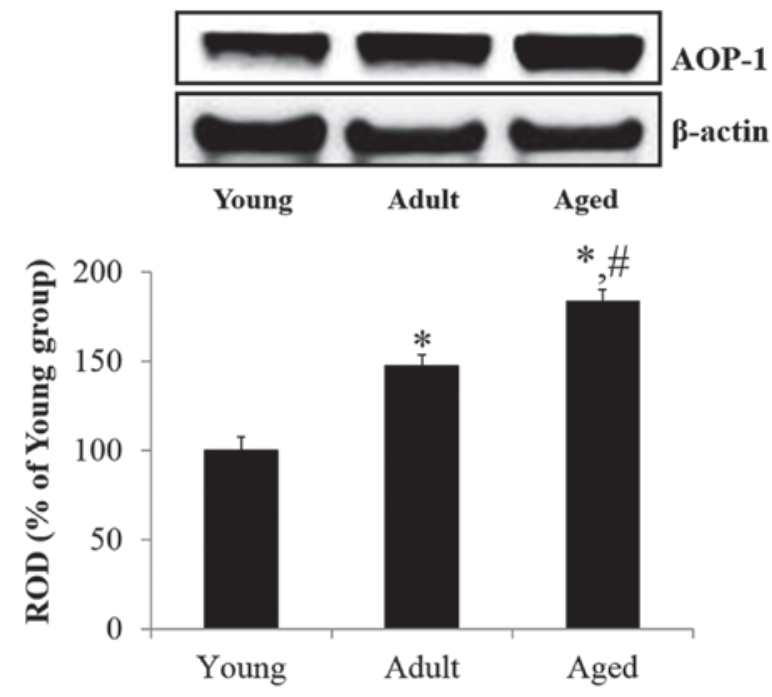

Figure 1. Western blot analysis of AOP-1 ( 25 kDa) in the hippocampus derived from the young, adult and aged groups. The ROD of the immunoblotting bands is shown as the percentage ( $\mathrm{n}=7$ /group; ${ }^{*} \mathrm{P}<0.05$, compared with the young group; ${ }^{\#} \mathrm{P}<0.05$, compared with the adult group). The data are presented as the mean \pm standard error of the mean. AOP-1, antioxidant-like protein-1; ROD, relative optical density.

streptavidin peroxidase complex (cat. no. SA-5004; 1:200; Vector Laboratories). A negative control test was performed using pre-immune serum instead of primary antibody in order to establish the specificity of the immunostaining. The negative control resulted in the absence of immunoreactivity in all structures.

A total of six sections with $90 \mu \mathrm{m}$ intervals per animal were selected to quantitatively analyze AOP-1 immunoreactivity. Digital images of the hippocampal subregions were captured with an AxioM2 light microscope (Carl Zeiss, Oberkochen, Germany) equipped with a digital camera (Axiocam; Carl Zeiss) connected to a PC monitor. According to our previous method (27), AOP-1 immunoreactivity was evaluated using digital image analysis software (MetaMorph 4.01; Universal Imaging Corp., Bedford Hills, NY, USA). The immunoreactivity was measured using a 0-255 gray scale system (white to dark signal between 255 and 0). AOP-1 immunoreactivity levels of experimental groups were calibrated with the background in the same section. Subsequently, the ratio of the immunoreactivity was calibrated as a percentage, with the young group designated as $100 \%$.

Statistical analysis. The data are expressed as the mean \pm standard error of the mean. The data were evaluated by a two-way analysis of variance using SPSS software (version 12.0; SPSS, Inc., Chicago, IL, USA) and the means were assessed using Duncan's multiple-range test. $\mathrm{P}<0.05$ was considered to indicate a statistically significant difference.

\section{Results}

AOP-1 protein level in the hippocampus. Western blot analysis demonstrated that the protein expression of AOP-1 was gradually and significantly increased in the hippocampus with 

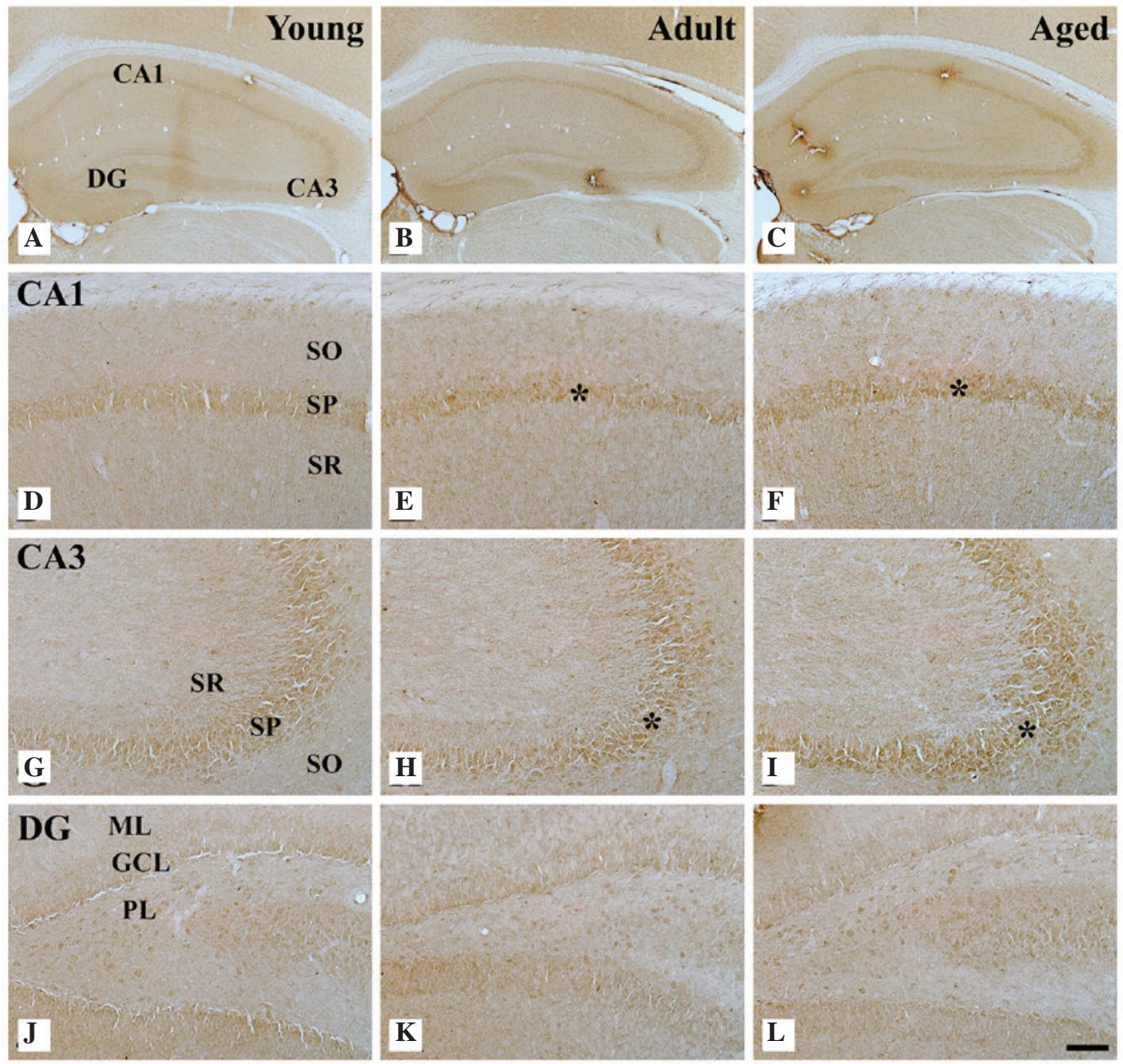

Figure 2. AOP-1 immunohistochemistry in the (A-C) hippocampus, (D-F) hippocampal CA1 region, (G-I) CA3 region and (J-L) dentate gyrus of the young (left), adult (middle) and aged groups (right). AOP-1 immunoreactivity is increased in the $\underline{\mathrm{SP}}$ (indicated by asterisks) of the CA1 and CA 3 region during the aging process. However, no marked difference was observed in AOP-1 immunoreactivity in the DG among all groups. Scale bar, $400 \mu \mathrm{m}$ (A-C) and $50 \mu \mathrm{m}$ (D-L). GCL, granule cell layer; ML, molecular layer; PL, polymorphic layer; SO, stratum oriens; SR, stratum radiatum; SP, stratum pyramidale; DG, dentate gyrus; AOP-1, antioxidant-like protein-1.

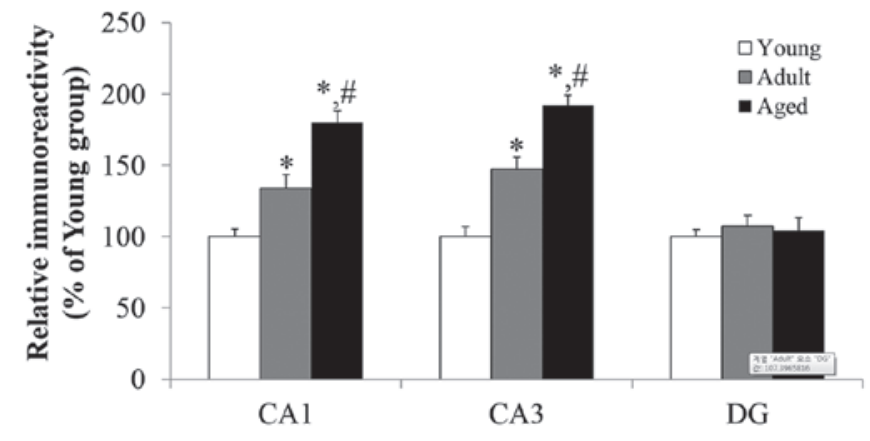

Figure 3. Relative immunoreactivity as a percentage of AOP-1 immunoreactive cells in the CA1 region, CA3 region and DG of the young, adult and aged groups ( $\mathrm{n}=7 /$ group; ${ }^{\mathrm{P}} \mathrm{P}<0.05$, compared with the young group; ${ }^{*} \mathrm{P}<0.05$, compared with the adult group). The data are presented as the mean \pm standard error of the mean. AOP-1, antioxidant-like protein-1; DG, dentate gyrus.

normal aging. The protein expression of AOP-1 in the adult hippocampus was significantly increased $(147.0 \%, F=9.914)$ compared with the young group. In the aged group, the protein expression of AOP-1 in the hippocampus was significantly higher $(124.7 \%, \mathrm{~F}=8.107)$ compared with that in the adult group (Fig. 1).

AOP-1 immunoreactivity in the hippocampus proper. Age-related changes in AOP-1 immunoreactivity (Fig. 2) in the hippocampus proper (CA1-3 regions) were found to be generally similar to the result of western blot analysis. In the hippocampus proper, weak AOP-1 immunoreactivity was primarily detected in pyramidal neurons of the stratum pyramidale of the young group (Figs. 2A, D, G and 3). In the adult group, AOP-1 immunoreactivity in the pyramidal neurons of the hippocampus proper was increased, compared with that in the young group (Figs. 2B, E, H and 3; F=34.407 in the $\mathrm{CA} 1$ region and $\mathrm{F}=25.579$ in the $\mathrm{CA} 3$ region, respectively). In the aged group, AOP-1 immunoreactivity was significantly increased in the pyramidal neurons of the hippocampus proper (Figs. 2C, F, I and 3; F=24.753 in the CA1 region and $\mathrm{F}=51.553$ in the $\mathrm{CA} 3$ region, respectively), compared with the young group. 
AOP-1 immunoreactivity in the dentate gyrus. In the young group, AOP-1 immunoreactivity was observed predominantly in granule cells of the granule cell layer and in polymorphic cells of the polymorphic layer (Fig. 2J). AOP-1 immunoreactivity in those cells of the dentate gyrus was not altered in the adult and aged groups, compared with that in the young group (Figs. 2K, L and 3).

\section{Discussion}

Our previous study demonstrated no marked age-related histopathological alterations in the hippocampus between adult and aged gerbils, as determined by terminal deoxynucleotidyl transferase dUTP nick end labeling staining and Fluoro-Jade B histofluorescence staining, although a marked activation of microglia was observed in the aged group (28). However, in the present, western blot analysis revealed that the protein expression of AOP-1 was significantly increased during the normal aging process. It can be postulated that the significant increase in AOP-1 protein level occurs due to the age-dependent increases in oxidative stress in the hippocampus.

Numerous previous studies have reported a significant increase in oxidative stress in the brain during the aging process $(14,15,20)$. On the basis of previous studies $(8,14-16,20)$, which demonstrated the age-related decrease in antioxidant enzymes in the brain, the basal levels of oxidative stress markers and the production of ROS in the hippocampus at various stages of aging was not measured in the present study. In particular, Selaković et al (20) reported that basal levels of certain types of oxidative stress, including superoxide anion production, superoxide dismutase activity and the index of lipid peroxidation, were significantly increased in the hippocampus of the 10-month-old gerbil, compared with those of the 3-month-old gerbil. It was also reported that the level of lipid peroxidation was increased and the levels of antioxidant enzymes, including catalase, superoxide dismutase and glutathione peroxidase, were decreased in the brain of aged rats compared with those of young controls (16). The authors suggested that the age-related decrease in antioxidant enzymes in the brain is due to deficient antioxidative enzyme defense against ROS (16).

The present study also found that AOP-1 immunoreactivity was significantly increased in the hippocampus proper, not in the dentate gyrus and among hippocampal subregions during the aging process. Therefore, it can be hypothesized that the age-dependent increase in AOP-1 occurs primarily in the hippocampus proper. However, it is difficult to explain exactly why the age-dependent increase in AOP-1 immunoreactivity occurs in the hippocampus proper and not in the dentate gyrus, since, to the best of our knowledge, this is the first study regarding the age-dependent change in AOP-1 protein expression in the hippocampus. Among the hippocampal subregions, the dentate gyrus is known as the most sensitive and vulnerable region during the normal aging process, whereas the hippocampus proper is relatively resistant (29). In addition, our previous study demonstrated that FoxO3a, which is strongly associated with the aging process and senescence $(30,31)$, was significantly decreased in the dentate gyrus, not in the hippocampus proper, of the aged gerbil hippocampus (26). On the basis of the present and other previous studies, it is likely that the expression patterns of biomarkers for brain aging are different according to brain areas. In this regard, enhanced AOP-1 expression in the hippocampus proper, not in the dentate gyrus, may be one of the possible mechanisms explaining why the dentate gyrus is more sensitive to oxidative stress compared with the other hippocampal subregions during the normal aging process.

In conclusion, the present study indicated that AOP-1 expression is significantly increased in the aged hippocampus, particularly in the hippocampus proper, during the normal aging process and suggests that age-related increases in AOP-1 may be a compensatory process against the elevation of oxidative stress in the aged hippocampus.

\section{Acknowledgements}

The present study was supported by a 2015 Research Grant from Kangwon National University (no. 520150344), the National Research Foundation of Korea (NRF) funded by the Ministry of Education, Science and Technology (no. 2010-0010580) and by the Basic Science Research Program through the NRF funded by the Ministry of Education (no. NRF-2014R1A1A2058440).

\section{References}

1. Shih SF, Wu YH, Hung CH, Yang HY and Lin JY: Abrin triggers cell death by inactivating a thiol-specific antioxidant protein. J Biol Chem 276: 21870-21877, 2001.

2. Feng Y, Liu DQ, Wang Z, Liu Z, Cao HQ, Wang LY, Shi N and Meng XM: AOP-1 interacts with cardiac-specific protein kinase TNNI3K and down-regulates its kinase activity. Biochemistry (Mosc) 72: 1199-1204, 2007.

3. Wonsey DR, Zeller KI and Dang CV: The c-Myc target gene PRDX3 is required for mitochondrial homeostasis and neoplastic transformation. Proc Natl Acad Sci USA 99: 6649-6654, 2002.

4. Fujii J and Ikeda Y: Advances in our understanding of peroxiredoxin, a multifunctional, mammalian redox protein. Redox Rep 7: 123-130, 2002.

5. Gallagher M, Bizon JL, Hoyt EC, Helm KA and Lund PK: Effects of aging on the hippocampal formation in a naturally occurring animal model of mild cognitive impairment. Exp Gerontol 38: 71-77, 2003.

6. He WB, Zhang JL, Hu JF, Zhang Y, Machida T and Chen NH: Effects of glucocorticoids on age-related impairments of hippocampal structure and function in mice. Cell Mol Neurobiol 28: 277-291, 2008.

7. Himeda T, Mizuno K, Kato H and Araki T: Effects of age on immunohistochemical changes in the mouse hippocampus. Mech Ageing Dev 126: 673-677, 2005.

8. Cardozo-Pelaez F, Brooks PJ, Stedeford T, Song S and Sanchez-Ramos J: DNA damage, repair, and antioxidant systems in brain regions: A correlative study. Free Radic Biol Med 28: 779-785, 2000.

9. Choi HS, Ahn JH, Park JH, Won MH and Lee CH: Age-dependent changes in expressions of Redd 1 and mTOR proteins in the gerbil hippocampus during normal aging. Mol Med Rep 13: 2409-2414, 2016.

10. Lee $\mathrm{CH}$ and Won $\mathrm{MH}$ : Increased dynamin-1 and -2 protein expression in the aged gerbil hippocampus. Cell Mol Neurobiol 34: 791-796, 2014.

11. Rutten BP, Korr H, Steinbusch HW and Schmitz C: The aging brain: Less neurons could be better. Mech Ageing Dev 124: 349-355, 2003.

12. Yang F, Chu X, Yin M, Liu X, Yuan H, Niu Y and Fu L: mTOR and autophagy in normal brain aging and caloric restriction ameliorating age-related cognition deficits. Behav Brain Res 264: 82-90, 2014.

13. Bagheri M, Joghataei MT, Mohseni S and Roghani M: Genistein ameliorates learning and memory deficits in amyloid $\beta$ (1-40) rat model of Alzheimer's disease. Neurobiol Learn Mem 95: 270-276, 2011 . 
14. Desai KM, Chang T, Wang H, Banigesh A, Dhar A, Liu J, Untereiner $\mathrm{A}$ and $\mathrm{Wu} \mathrm{L}$ : Oxidative stress and aging: Is methylglyoxal the hidden enemy? Can J Physiol Pharmacol 88: 273-284, 2010.

15. Finkel T and Holbrook NJ: Oxidants, oxidative stress and the biology of ageing. Nature 408: 239-247, 2000.

16. Haider S, Saleem S, Perveen T, Tabassum S, Batool Z, Sadir S, Liaquat L and Madiha S: Age-related learning and memory deficits in rats: Role of altered brain neurotransmitters, acetylcholinesterase activity and changes in antioxidant defense system. Age (Dordr) 36: 9653, 2014.

17. Aydin AF, Coban J, Doğan-Ekici I, Betül-Kalaz E, Doğru-Abbasoğlu S and Uysal M: Carnosine and taurine treatments diminished brain oxidative stress and apoptosis in D-galactose aging model. Metab Brain Dis 31: 337-345, 2016.

18. Qu Z, Zhang J, Yang H, Huo L, Gao J, Chen H and Gao W: Protective effect of tetrahydropalmatine against d-galactose induced memory impairment in rat. Physiol Behav 154: 114-125, 2016.

19. Tchantchou F, Chan A, Kifle L, Ortiz D and Shea TB: Apple juice concentrate prevents oxidative damage and impaired maze performance in aged mice. J Alzheimers Dis 8: 283-287, 2005.

20. Selaković V, Rauš Balind S, Radenović L, Prolić Z and Janać B: Age-dependent effects of ELF-MF on oxidative stress in the brain of Mongolian gerbils. Cell Biochem Biophys 66: 513-521, 2013.

21. Kan H, Hu W, Wang Y, Wu W, Yin Y, Liang Y, Wang C, Huang D and $\mathrm{Li} \mathrm{W}$ : NADPH oxidase-derived production of reactive oxygen species is involved in learning and memory impairments in 16-month-old female rats. Mol Med Rep 12: 4546-4553, 2015.

22. Liao J, Xia X, Wang GZ, Shi YM and Ge JW: Naotaifang extract treatment results in increased ferroportin expression in the hippocampus of rats subjected to cerebral ischemia. Mol Med Rep 11: 4047-4052, 2015.

23. Sasaki $\mathrm{K}$ and Yoshizaki F: Investigation into hippocampal nerve cell damage through the mineralocorticoid receptor in mice. Mol Med Rep 12: 7211-7220, 2015.
24. Xu Y, Liu Z, Song X, Zhang K, Li X, Li J, Yan X, Li Y, Xie Z and Zhang H: Cerebralcare Granule ${ }^{\circledR}$ attenuates cognitive impairment in rats continuously overexpressing microRNA-30e. Mol Med Rep 12: 8032-8040, 2015.

25. Cheal ML: The gerbil: A unique model for research on aging. Exp Aging Res 12: 3-21, 1986.

26. Park JH, Lee CH, Yoo KY, Choi JH, Hwang IK, Lee JY, Kang IJ and Won MH: FoxO3a immunoreactivity is markedly decreased in the dentate gyrus, not the hippocampus proper, of the aged gerbil. Exp Gerontol 46: 836-840, 2011.

27. Lee CH, Park JH, Cho JH, Kim IH, Ahn JH, Lee JC, Chen BH, Shin BN, Tae HJ, Bae EJ, et al: Effect of oenanthe javanica extract on antioxidant enzyme in the rat liver. Chin Med J (Engl) 128: 1649-1654, 2015

28. Lee CH, Yoo KY, Choi JH, Park OK, Hwang IK, Kim SK, Kang IJ, Kim YM and Won MH: Neuronal damage is much delayed and microgliosis is more severe in the aged hippocampus induced by transient cerebral ischemia compared to the adult hippocampus. J Neurol Sci 294: 1-6, 2010.

29. Small SA, Chawla MK, Buonocore M, Rapp PR and Barnes CA: Imaging correlates of brain function in monkeys and rats isolates a hippocampal subregion differentially vulnerable to aging. Proc Natl Acad Sci USA 101: 7181-7186, 2004.

30. Kyoung Kim H, Kyoung Kim Y, Song IH, Baek SH, Lee SR, Hye Kim J and Kim JR: Down-regulation of a forkhead transcription factor, FOXO3a, accelerates cellular senescence in human dermal fibroblasts. J Gerontol A Biol Sci Med Sci 60: 4-9, 2005.

31. Li M, Chiu JF, Mossman BT and Fukagawa NK: Down-regulation of manganese-superoxide dismutase through phosphorylation of FOXO3a by Akt in explanted vascular smooth muscle cells from old rats. J Biol Chem 281: 40429-40439, 2006. 